\title{
The permanently charged lidocaine analogue QX222 acts as a blocker from the intracellular side and as an inactivation modulator from the extracellular side in a mutant $\mathrm{Na}_{\mathrm{v}} 1.4$ channel
}

\author{
Péter Lukács, René Cervenka, Xaver Koenig, Agnés Mike, Touran Zarrabi, Karlheinz Hilber, Hannes Todt
}

From 16th Scientific Symposium of the Austrian Pharmacological Society (APHAR)

Vienna, Austria. 25-27 November 2010

\section{Background}

QX222 is a quaternary amine analogue of lidocaine, which, unlike lidocaine, is permanently charged. Lidocaine has its binding site in the internal vestibule of the voltage-gated sodium channel. Due to the hydrophobic nature of its uncharged form, lidocaine reaches the binding site by passing through the membrane, QX222 can reach this binding site only by a hydrophilic pathway, presumably through the channel protein. However, such a pathway has been reported only in the heart-type sodium channel $\left(\mathrm{Na}_{V} 1.5\right)$ and some mutants of other sodium channels. Notably, mutations at site 1575 in the skeletal muscle-type sodium channel $\left(\mathrm{Na}_{\mathrm{V}} 1.4\right)$ open an access pathway from the external side. In this study we tested the properties of QX222 block on the mutant I1575E.

\section{Methods}

All measurements were done in tsA201 cells transiently transfected with the $\mathrm{Na}_{V} 1.4$ sodium channel $\alpha$ subunit, cotransfected with $\beta 1$ sodium channel subunit. Currents were recorded by patch-clamp technique in whole-cell configuration.

\section{Results}

Both $500 \mu \mathrm{M}$ lidocaine and $500 \mu \mathrm{M}$ QX222 shifted the half-point of steady-state slow inactivation to hyperpolarized potentials in I1575E if applied from the extracellular side. However, only lidocaine significantly shifted the half-point of steady-state fast inactivation. Intracellular application of QX222 resulted in a quick block of sodium current, indicating that the drug entered the channel, but the hyperpolarizing shift of steady-state fast inactivation was still not present. In addition, with intracellular application of QX222, the strong hyperpolarizing shift in steady-state slow inactivation disappeared.

\section{Conclusions}

These results suggest that the binding site for usedependent block is in the inner vestibule of the channel, that fast inactivation is modulated only by the hydrophobic form of local anaesthetics, and that the binding site for modulation of slow inactivation is only accessible form the extracellular side of the channel.

\section{Acknowledgements \\ This work was supported by grants P21006-B11, P17509-B11, P13961-B05 from the FWF.}

Published: 16 November 2010

doi:10.1186/1471-2210-10-S1-A24

Cite this article as: Lukács et al:: The permanently charged lidocaine analogue QX222 acts as a blocker from the intracellular side and as an inactivation modulator from the extracellular side in a mutant $\mathrm{Na}_{\mathrm{v}} 1.4$ channel. BMC Pharmacology 2010 10(Suppl 1):A24.

\footnotetext{
* Correspondence: hannes.todt@meduniwien.ac.at Institute of Pharmacology, Center of Physiology and Pharmacology, Medical University of Vienna, 1090 Vienna, Austria
} 